\title{
Contre l'idiotie hobo et bohémienne ou le bovarysme selon Widmer
}

\section{Against the hobo and bohemian idiocy or the Bovarianism according \\ Widmer}

Simon Harel $^{1}$

Submetido em 18 e aprovado em 20 de março de 2017.

Résumé: D’après Kingsley Widmer, l'enthousiasme suscité par la tendance bohème de la Beat Generation, qui s'est popularisée après la Seconde Guerre mondiale, procède d'une vision conformiste du monde. En effet, la description de la bourgeoisie effrayée par la violence contraignit ses adeptes à exprimer leurs sentiments par d'autres moyens. La tendance bohème est un bovarysme, un provincialisme adopté par des personnalités dans certaines régions. En reproduisant l'intensité propre à ces êtres désespérés, elles transforment finalement la rébellion et la solitude en une tendance banale. Kingsley Widmer est un essayiste méconnu et un professeur. En 1956, il a commencé à enseigner au State College de San Diego, qui devint ensuite la San Diego State University (Université d'État de San Diego). Il a également défendu une thèse à l'Université de Washington. Il fut Président des Étudiants du Mouvement pour une Société Démocratique à l'Université d'État de San Diego. Il a écrit des textes sur de nombreux sujets divers, allant d'Henry Miller à Paul Goodman. Le ton des essais de Widmer est le plus souvent sarcastique, voire agressif. Généralement, ses écrits dénotent un vif intérêt pour l'anarchie. Ses travaux n'ont pas été réédités et ses œuvres sont malheureusement tombées dans l'oubli.

Mots-clés: Bohémien. Bourgeoisie. Contre-Culture. Consommation. Vagabondage. Années Soixante. États-Unis.

\begin{abstract}
According to Kingsley Widmer, the enthusiasm surrounding the bohemian current of the Beat movement, which gained popularity after the Second World War, stems from a conformist view of the world. In this sense, the description of the bourgeoisie frightened by violence forced them to channel their feelings through other means. The realm of bohemian is a bovarysm, a provincialism of the public figures of a given province whom, using the intensity inherent to these desperate people, shape rebellion and solitude into one common realm. Kingsley Widmer is an unknown essay writer and professor. In 1956, he started to teach at San Diego State College, which later became San Diego State University. He also defended a thesis at the University of Washington. President of the Students for a Democratic Society movement at San Diego State University, he has written texts on a plethora of topics, ranging from Henry Miller's works to Paul Goodman's. The tone of Widmer's essays is often sarcastic and even aggressive. Generally, his writings show a keen interest in anarchy. Widmer's texts have not been reedited and his works have unfortunately been forgotten.
\end{abstract}

Keywords: Bohemian. Bourgeoisie. Counter culture. Consumption. Vagrancy. Sixties (1960s). United States of America. 
L'idiot, c'est celui qui, condamné au principe de la clarté, de l'expression cohérente de soi, s'en remet à l'idiosyncrasie du réel. C'est dire que la figure de l'idiot ${ }^{2}$ permettrait de poursuivre la réflexion sur ces «sous-sujets» que sont autant les sans-abris que les sujets souffrant de maladie mentale, mis à la rue, désinstitutionnalisés, et qui incarnent de nouvelles formes d'errances urbaines.

Peu d'ouvrages ont été consacrés à l'idiotie. Parmi les références éparses, il y a $L e$ réel. Traité de l'idiotie (1986) de Clément Rosset qui aborde ces questions d'un point de vue philosophique ou L'idiotie. Art, vie, politique - méthode (2003) de Jean-Yves Jouannais qui traite de l'idiotie dans le domaine artistique, en mettant l'accent sur les relations entre l'idiotie et ce que j'appellerais l'art prémédité. Jouannais, dans la foulée des spécialistes de l'art brut, tente en effet de mieux comprendre de quelle manière les énonciations a priori marginales (celles des «fous», des «exclus» de l'existence) réussissent, par le biais de la création, à soutenir non pas tant une réflexion qu'une création authentique dont l'idiosyncrasie, forme pérenne de l'idiotie, serait le dénominateur commun.

Jean-Jules Richard dans Le Journal d'un hobo (1965) participe d'un fantasme puissant avec sa vision euphorique du hobo, une vision qui entraîne avec elle des rêveries de libération. C'est en soi une idiotie puissante et salvatrice que la version nocturne de Ferron $(1965 ; 1979)$ contient aussi, avec son espoir de liberté pour l'ensemble des Québécois francophones. Néanmoins, de telles idioties bohémiennes ne sont pas toujours valorisées quand il s'agit de les connecter avec un esprit de concertation nationale, tel qu'il existe aussi dans les années 1960. C'est-à-dire l'esprit d'êtres qui, à la suite des hobos du Dust Bowl ${ }^{3}$, envahissent les villes du Nord, les métropoles de la Côte-Ouest, pour scander, sur fond de jazz et de percussions, l'oralité dite primitive d'une poésie décidément urbaine. Ce sont les Beats. Ferlinghetti, Corso, Snyder, tous ces poètes qui incarnaient autant de portes-voix d'une urbanité dissonante se percevaient comme de nouveaux bluesmen de la modernité, des sujets certes blancs, mais capables de se déhancher, de faire vibrer le monde dans lequel ils vivaient.

Or, le cosmopolitisme se fait labyrinthique. La voix des autres peut être entendue sans que le sujet fixe du regard son vis-à-vis, son alter ego: la salle des pas perdus me revient alors en mémoire en ce qu'elle représente, au cœur de la ville, ce cosmopolitisme 
labyrinthique, une forme d'évitement, assez semblable à la superficialité des postures bohémiennes qui circonscrivent le caractère factice de marginalités qui, cette fois, s'en remettent au principe de la gentrification et de la distinction symbolique.

Comme toute médaille a son revers, il existe donc l'opposé de l'été, le contraire d'une joie, d'une idiotie. Cet envers du décor s'appuie sur une diatribe concernant l'esprit de bohème dans sa version européenne d'abord, puis américaine. Une telle diatribe transforme l'esprit de liberté en actes vains. C'est le cas de The Literary Rebel (1965) de Kingsley Widmer qui nous permet de revenir à l'évolution de l'esprit bohème à l'esprit bobo, en passant par la singularité qu'incarne le hobo américain.

La première publication de The Literary Rebel date de mars 1965, autrement dit de l'hiver 1965 - la réalité rattrape ici la symbolique saisonnière que j'ai mise en place en parlant d'été, sans que je l'aie souhaité pourtant, je l'affirme. Une seconde édition verra le jour un an plus tard, en 1966. Avec The Literary Rebel, Kingsley Widmer perçoit la représentation de la figure du bohémien dans la culture américaine du $19^{\mathrm{e}}$ siècle, puis lors de sa formalisation urbaine, par exemple, à Greenwich Village, au cours du 20e siècle (WIDMER, 1965, p. 4-15). Il la critique vertement. Pour en saisir toutes les implications, il convient néanmoins de concevoir la constellation que l'histoire dessine à grands traits : le bohème le hobo le bobo. Cette constellation se pense mieux en croisant les disciplines, comme la géographie (avec Anne Clerval), la sociologie (avec Nels Anderson) et l'histoire (Jean-François Wagniard). Ensuite, il sera loisible de mieux voir en quoi, dans le contexte des années 1960, Widmer propose une réflexion d'une grande vivacité à l'égard de ces prétentions de poètes et d'écrivains qui, par le biais de leurs écrits, pensent connaître mieux que quiconque le souffle de la rébellion, l'expression de l'anti-pensée.

Lire un auteur comme Widmer, c'est nous permettre de faire la jonction entre l'Europe et l'Amérique à partir de la figure du bohémien, de revenir aux sources de ce qu'est le hobo ou du moins de ce qui le précède, d'en revenir à l'idiotie. Cela permet de saisir en quoi la fantasmatique du hobo telle que Richard nous en dresse un portrait piquant se télescope avec la notion de bohème, toujours fascinante, peut-être plus encore en ces 
temps difficiles. Car il y a des questions à soulever, celle de la ressemblance phonétique entre hobo et bobo n'est pas la moindre, et l'on peut se demander avec la géographe Anne Clerval ce qu'auraient en commun ces deux «personnages idéaux-typiques» (CLERVAL, 2005 , s. p.). En effet, d'un côté, il y a un sans-domicile qui saute de train en train à la recherche de travail et de nourriture, d'expériences fortes aussi, de l'autre, un bourgeois bohème dont l'oxymore pose de suite la condition complexe - voire contradictoire -, puisqu'il est à la fois dans le rejet et l'acceptation de la société.

Quel est alors le dénominateur commun entre le hobo du début du $20^{\mathrm{e}}$ siècle et le bobo apparu dans la seconde moitié du même siècle? C'est la bohème. On a l'habitude de faire preuve de romantisme à l'égard de l'itinérance en général (associée plus ou moins à la bohème), comme s'il s'agissait toujours d'une trajectoire choisie. On a aussi l'habitude de faire valoir qu'elle est un mode de contestation du discours social, de son autorité. Kerouac a marqué durablement les esprits. Ce faisant, l'itinérance devient l'une des composantes d'un discours qui prend sa source dans l'univers bohémien, tel qu'il est décrit dans le discours social du $19^{\mathrm{e}}$ siècle, d'Henry Murger à Colin Wilson, à Paris ou à Londres ${ }^{4}$, et tel qu'il est encore vécu de nos jours. En effet, les nouveaux bohémiens ou, selon le terme convenu en français, les «bobos», sont légion dans les villes d'Occident, comme si la métropolisation du monde engendrait, sous l'aspect de périphéries acceptables, l'existence de populations marginales qui contribuent à redéfinir la notion de culture et d'espace public.

Pour qui s'intéresse à la mise en scène du bohémien, les nominations s'avèrent très variées. Si l'on s'en tient au domaine américain, The White Negro: Superficial Reflections on the Hipster (1957) est un texte particulièrement important des années 1950, dans lequel Norman Mailer, écrivain d'une verve remarquable, d'un appétit de rébellion inassouvissable, met en relation la colère des Afro-américains et l'émergence d'une culture «blanche» de la dissidence: le beat, le hippie, voire le yuppie. Autrement dit, la politique est au cœur de la réflexion de la bohème et, en l'occurrence, des conflits interraciaux. Un Herbert Gold, écrivain majeur, malheureusement méconnu aujourd'hui, écrit quant à lui un article qui a pour titre The Beat Mystique (1958). Quant au poète Kenneth Rexroth, il est l'auteur du fameux San Francisco's Mature Bohemians (1957). 
Mais si l'on décide de remonter le temps, c'est en 1905 que Georges Plekhanov écrit Art and Society: a Marxist View, un texte substantiel mettant en relation l'attitude bohémienne d'un point de vue marxiste et le rôle joué par les formes de l'art dans la société. Toutes ces références nous permettent de concevoir que l'univers sémantique du bohémianisme est extensible et que les liens entre hobo et bohème sont plus forts qu'il n'y paraît de prime abord, ce qui fait relire Davies d'abord, Richard ensuite, en fonction de cette donnée liée à la création.

Se dessine ainsi une constellation : bohème, hobo et bobo. Et il ne faudrait pas croire que les trois univers ne se rencontrent jamais ou que les hobos ne sont qu'un accident de parcours entre le bohémien et sa version actuelle boboïsée. C'est en effet l'une des leçons à retenir de l'étude d'Anderson, qui démontre qu'à Bughouse Square, à Chicago, la rencontre peut avoir lieu entre la bohème et la hobobohème: «Pour le hobo qui réfléchit, même si sa réflexion ne va pas loin, la partie basse de la rive Nord est une grande source de réconfort. Là, il trouve des gens à qui il peut parler et qu'il est prêt à écouter. (...) Beaucoup sont là pour échapper à la laideur de la vie dans d'autres quartiers de la Hobobohème.» (ANDERSON, 2011, p. 67). C'est l'un des liens, aussi, qui se tissent à la lecture de la diatribe de Widmer, lequel s'oppose, entre autres, à la définition du hobo selon Mailer. Il ne voit pas dans le phénomène une dissidence réelle mais plutôt une forme de jeu qui déforme ce qu'est la bohème.

La bohème qui retient mon attention, ce n'est pas celle de notre époque. Celle qui est la nôtre n'a de valeur à mes yeux que mise en perspective avec son histoire propre qui la fait naître dans un contexte, puis la transforme et l'acculture ailleurs. Ainsi l'élite qui émerge à la toute fin du $20^{\mathrm{e}}$ siècle est-elle loin de ce que fut le bohémien du siècle précédent, même si elle souhaite en reprendre les éléments définitoires: scepticisme envers la société, dimension artistique prononcée et attitude qui coïncide à un moment donné avec une dégaine nonchalante, ce que l'on appelle dans le Greenwich Village des années 1930 le «cool», tandis que Nels Anderson essaie de radiographier ce qu'est le hobo à l'aune de la sociologie. Cette nonchalance étudiée traduit le scepticisme de l'artiste 
à l'égard de cette animation frénétique qu'il voit s'exercer au cœur des grandes villes d'Europe et d'Amérique, mais là encore, elle n'est pas à l'origine de ce qu'on appelle le « ohémien». En conséquence de quoi, le bohémien n'est pas non plus celui qui appartient à cette frange de la jeunesse américaine clamant à corps et à cri son progressisme dans les années 1960, tandis que Richard publie Journal d'un hobo (1965). C'est celui qui s'origine en France, à la fin du $19^{\mathrm{e}}$ siècle. Celui qui est artiste.

Certes, tous les bohémiens de Paris ne furent pas artistes, ce serait naïf de le croire, mais certains oui, ainsi que le rappelle Clerval: «[L]a bohème renvoie à une vie marginale, sans argent et parfois sans toit, qui était le lot de ces artistes d'abord non reconnus [Nerval, Rimbaud], notamment parce qu'ils critiquaient ouvertement les valeurs bourgeoises.» (CLERVAL, 2005, s.p.) (Bref, on en revient à un avant-hobo pour mieux saisir en quoi, en définitive, à un moment donné d'une construction fantasmatique, il y a un rapprochement entre le bohémien et le hobo. Et ce rapprochement, Anderson a pu le prouver au siècle suivant, en évoquant une certaine proximité entre les deux catégories distinctes - proximité dans le lieu et dans la langue. Il a pu le prouver, aussi, en s'intéressant à la forme artistique privilégiée par les hobos: l'écriture.

Cela contraste avec ce qu'on sait par ailleurs de la majorité des sans-abris lorsqu'on lit les études sociologiques qui leur sont consacrées: même s'ils disent beaucoup, fabulent, racontent, ils n'ont pas pour propriété singulière d'être des personnes qui font un exercice mesuré du langage. Leur prise de parole est souvent zigzagante, hachurée ; elle s'affaisse, se transforme tout à coup en borborygme. Que faire de ce discours qui a toutes les apparences de la confusion, du délire, de la pâte verbale sans relief, en d'autres termes, que faire de ce discours qui n'en est pas un? Pas grand-chose.

En revanche, il y a une frange de sans-abris à la prise de parole claire, assumée. Une parole sur la place publique, de vive voix, par exemple William Davies avec Carnets d'un hobo en 1908, ou par journaux interposés, que l'on songe au Hobo News ou à Industrial Solidarity, dans les années 1920 aux États-Unis - c'est-à-dire finalement, dans des espaces d'expression radicalisée. Ils prennent la parole, la diffusent, lisent celle des autres.

C'est donc que le hobo pense, écrit et lit. Davies l'a prouvé. Et Anderson précise combien, par rapport aux sédentaires, le vagabond et le hobo sont des lecteurs, même 
s'ils ne choisissent pas vraiment leur lecture et prennent ce qui leur tombe sous la main. De la lecture à l'écriture, le pas est souvent franchi: «Le hobo qui lit s'essaie tôt au tard à l'écriture. Un nombre étonnamment élevé de hobos réalisent leur ambition de se faire imprimer» précise Anderson avant d'ajouter que les «écrivains hobos ne s'occupent pas seulement de lettres» car certains «ont l'ambition de devenir romanciers, essayistes, et même auteurs dramatiques» et ils possèdent même des manuscrits, qu'ils gardent précieusement sur eux, où qu'ils aillent (ANDERSON, 2011, p. 248).

Le télescopage entre hobo et bohème advient donc par l'entremise d'un mot qui sert de lien, de liant : la création. Il n'est pas incompatible de penser tout en marchant, de fabuler tout en déambulant, de rêver même si la nuit est froide et que les os nous font mal. Le narrateur du Journal d'un hobo s'invente lui-même, se façonne à partir de sa double identité jusqu'à la fusionner en une sorte de mise en abyme du travail de l'écrivain luimême, et ce travail-là contient une charge érotique forte. C'est la création d'une identité, comme dans toute littérature des frontières ${ }^{5}$. Et la lecture de Richard nous éclaire sur le sujet, en mettant l'accent sur la contestation (rappelons-nous la Marche de la faim), la critique de l'autorité et la liberté sexuelle. Et, en le faisant, de manière poétique.

On peut donc, à propos de la création, mettre en avant des œuvres comme celle de Kerouac et de London, bien entendu, et en même temps celle de Richard, à la fois dans son prolongement et sa connexion totalement affirmée entre hobo-bohémien-création (littéraire/identitaire). On pourra même ajouter que, dans le Hobo News, publié de manière mensuelle, il existait une rubrique «Coin des poètes» permettant à certains hobos de s'exprimer librement et de manière poétique ${ }^{6}$. On touche ici à un point très particulier de la fiction du hobo telle qu'elle va se mettre en place en rejoignant une vision positive de l'artiste bohème de la fin du 19e siècle, capable de transformer la réalité en œuvre et aussi de jouer un rôle.

Cette valorisation par l'esthétique date donc de la fin du $19^{\mathrm{e}}$ siècle et elle vient du monde artistique lui-même. L'historien Jean-François Wagniart note à ce sujet une proximité symbolique entre le vagabond et l'artiste. Loin de faire du vagabond une figure 
déchue (Zola), certains artistes de la fin du 19e siècle peuvent aller jusqu'à souhaiter lui ressembler et conçoivent alors l'errance comme une forme ultime de la liberté (Bloy, Rictus ou Philippe) tandis que d'autres contribuent tant que faire se peut à valoriser celui dont on pense le pire (Mirbeau, Maupassant ou Vallès). De sorte qu'un certain nombre d'écrivains, en se projetant dans cette condition de vagabond de l'esprit, «se veulent les représentants de cette culture populaire singulière, de cet imaginaire qui fait du vagabond une figure authentique de l'illégalisme populaire et un défi perpétuel aux lois» (WAGNIART, 1999, p. 98). C'est dire qu'une fantasmatique puissante, dans la France de cette époque, crée une correspondance cherchant à valoriser une figure rendue encore plus extrême par la plume, afin de servir cette «authenticité» dont il est question, authenticité qui s'arrime à la notion de liberté - les vagabonds ne sont pas contaminés par la société serait l'idée sous-jacente.

À ce point de la réflexion, on ne peut que se poser la question de Wagniart: «audelà de sa diffusion ou de son impact, ce contre-feu littéraire est-il capable de produire cette contre-image qui influence positivement la vision générale de l'errance, afin de rendre une identité au vagabond noyé dans le monde des délinquants?» (WAGNIART, 1999, p. 99). Car, à la fin du 19e siècle, l'écrivain bohème, le poète clochard, de même que le révolutionnaire (tel que la société le fantasme) sont «des figures aussi honnies que celle de l'errant» (WAGNIART, 1999, p. 97). Autrement dit, il y a une forme de diabolisation en même temps qu'il y a une promotion de cette figure singulière. C'est que le vagabond fait peur, qu'il incarne même toutes les peurs, y compris et surtout celle du chômage en France. Cette peur, elle est aussi présente dans la vision de la société américaine. À Chicago dans les années 1920, le chômage conduit à des chiffres effrayants - 30000 sansdomiciles (homeless men) en été, 60000 en hiver, 75000 en période de chômage. Surtout, en période de récession, les gens qui subissent la perte d'emploi ont peur de croiser ces miséreux qui, il faut le dire, leur rappellent leur propre condition - à travers un miroir déformant, poussiéreux, piqué.

Entre 1920 et 1960, en terre américaine, il va s'opérer un changement considérable de point de vue: le hobo sera en partie valorisé. D’abord, les autorités le tolèrent et les journaux leur offre une belle visibilité. Mais la réelle modification tient à cette superposition 
d'une figure ancienne, celle de la bohème venue d'Europe, à une réalité économique terrible. Chez Wagniart, on trouvera un élément de réponse sur ce que l'artiste bohème a apporté au vagabond, et dont le hobo américain héritera, à savoir l'idée selon laquelle «le vagabond n’est ni forcément délinquant ni forcément criminel» (WAGNIART, 1999, p. 100).

Le hobo peut être poète et il l'a prouvé. Un poète de la révolte, un chantre de la liberté qui, parfois, met en rimes les choses les plus cruelles, les plus dures. Comme l'artiste français avant lui, il observe de loin la foule animée et hétéroclite. Ce plaisir esthétique implique forcément une forme de désabusement, puisque le regard se veut détaché. Ainsi Richard recrée-t-il une vision en hauteur de ce que le hobo peut voir, un peu à la manière de l'artiste dans sa ville:

La cour ferroviaire, comme une pieuvre, s'étend dans toutes les directions. De puissants réflecteurs font ressortir ces grosses boîtes que sont les wagons de fret : mille hommes, mille chevaux. Abouchés les uns aux autres, de mystérieux pouvoirs les font circuler. Ils glissent dans la nuit et c'est étrange: on dirait des danseuses de ballet en grandes robes. La nuit transpire de chaleur (WAGNIART, 1999, p. 95).

Le bohémien français puis le hobo américain peuvent donc se rejoindre lorsque, détachés des contingences industrielles et financières du monde, ils incarnent un point de vue transcendant, une autorité narrative qui, sans qu'elle corresponde au discours des références religieuses et monarchiques, ce que Jean-François Lyotard décrit sous le titre de «Grand Récit», n’en est pas moins surélevée, rehaussée. Il s'agit de voir le monde de haut, la foule cosmopolite, l'univers des passants, puis l'énergie machinique d'un mouvement perpétuel que la ville industrielle du $19^{\text {e }}$ siècle semblait promettre. C'est exactement cette vision que l'on trouve donc chez Richard.

De plus, en ce qui concerne le dire poétique, Richard va beaucoup plus loin que Davies, dont les carnets publiés fonctionnent comme la pré-histoire du Journal d'un hobo, c'est-à-dire le moment où le hobo devient écrivain mais sans élan franchement poétique. Richard, lui, arrache la réalité de la terrible condition du hobo, afin de la transformer en une substance poétique aux accents céliniens:

Des accidents se produisent. De beaux jeunes gens n'ont plus qu'une jambe, qu'un bras, qu'une main pour enlacer. Les roues sur 
le rail sectionnent la chair comme des brins de foin. La sève s'en écoule et le rail se peint en rouge pour quelques mètres et c'est tout. Du bruit des roues, le rail se bouche les oreilles pour ne pas entendre les plaintes. Quelqu'un, plus tard, ramasse la dépouille. Adresse inconnue. Gibier d'assistance publique. Cochon d'Inde pour les internes (RICHARD, 1965, p. 96).

Dans la même veine, qui joue sur l'anthropomorphisation et l'animalisation du train, objet de peur et moyen de se sentir libre pour le hobo:

Je surprends le Fauve à ramper sous un wagon. Il ne faut pas tenter le sort ainsi. La locomotive accoupleuse travaille à préparer un long ruban de ferraille, mais elle est aveugle et ses anneaux sont ceux d'une vipère. Écraser un membre ou deux n'est rien pour elle (RICHARD, 1965, p. 146).

Petit à petit, le fantasme du hobo, dans sa version positive, se modifie, au gré des sociétés qui le fantasment, et en se télescopant avec la notion de bohème d'où il est issu en définitive. Mais qu'en est-il d'une version plus contrastée? Moins lumineuse, moins poétique, moins sereine? Une œuvre, The Literary Rebel de Kingsley Widmer, va nous aider à en saisir les contours. Elle incarne à elle seule l'envers de l'été 1965.

Selon Widmer, le bohémianisme est une forme d'ascèse qui consiste à se tenir en retrait de la vie sociale, à exprimer le plus vif mépris envers les bourgeois si actifs dans l'univers de la modernité mécanique. Pour mieux le faire comprendre, l'essayiste se réfère au style cher à Diogène, le philosophe grec: il s'agit de contester la parole des rois et des maîtres des armées et de se promener nu dans la rue, sans respecter les règles minimales d'hygiène. Dans la pensée occidentale, Diogène symbolise cette apparition de philosophes va-nu-pieds, de personnages forts en gueule et autres cyniques qui s'opposent au pouvoir de l'identité propre et à la foule des semblables qui s'empresse de rehausser et d'instaurer celui qui veut coûte que coûte devenir prêtre, chef d'état, chef des armées. Avec Widmer, le voilà associé malgré lui au bohémien - mais peut-être cela ne lui aurait pas déplu.

Ceci étant posé, quittons l'Antiquité et posons-nous la question du pouvoir de contestation des bohémiens au cœur de la société de consommation, cette invention typiquement américaine. Les bohémiens sont en mesure de contester l'émergence de 
cette société avec un sens de la dérision remarquable : alors que tous veulent monter dans l'échelle sociale, ils font savoir qu'ils se contentent de vivre au cœur de l'univers des arts et des lettres, sans aucune considération pour la valeur d'échange du capital qui leur permettrait de prétendre à un nouveau statut social.

Le point de départ de la réflexion de Widmer consiste donc à mettre de côté la représentation stéréotypée et folklorique du hobo pour préférer en revenir la figure du vagabond en général, dont l'appartenance au vaudeville et au monde du burlesque ne peut être sous-estimée:

Such a pair of tramps wander, with relevant anachronism, into the poetic drama in Samuel Beckett's Waiting for Godot. There they combine nihilistic pathos and buffoonish mockery in one of those peculiarly contemporary attempts to get outside the comic-tragic teleology of the past. Wherever Beckett may have acquired his two ex-hobos, they seem comprehensible as a mannerist version of the vaudeville Chaplin cartoon little tramps (WIDMER, 1965, p. 108).

Chez Chaplin, le vagabond n'est pas une figure héroïque. C'est un être déboîté, ayant de toute évidence perdu le vernis de sociabilité qui permet de créer les conventions nécessaires pour que l'illusion d'une vie commune soit maintenue. Son apparente gaieté, en tous les cas la spontanéité de son geste, se traduit par une mélancolie insondable. Aux yeux de Widmer, dans En attendant Godot (écrite en 1948; publiée en 1952) de Beckett, cette dévaluation du vagabond s'accentue encore, entraînant une chute sans espoir de rétablissement. Estragon et Vladimir, les deux personnages principaux de la pièce, sont deux vagabonds qui habitent un univers métaphysique sans profondeur, un lieu qui n'a plus la forme du réceptacle, de cette nasse, de ce claustrum en mesure d'accueillir le sujet, de lui offrir une juste place. La camaraderie de ses deux personnages tient lieu d'étayage, de béquille, de manière à ce que ladite relation à autrui, qui prend l'aspect d'une liturgie idiote, soit une fois pour toute mise en cause.

Les rebelles, que représentent Estragon et Vladimir et aussi Pozzo et Lucky dans En attendant Godot, se contentent de faire intervenir, au nom de ce que j'appelle l'itinérance, une réversibilité de prise de parole, un jeu sardonique, une parodie violemment assénée 
de ces rôles qui nous font être sujets dans le théâtre de la vie ${ }^{7}$. Une esthétique de la laideur est ici mise en place, un art de l'itinérance qui consiste à se montrer tel qu'on est, à ne pas jouer sur les apparences. De même, les déplacements des sujets dans l'espace sont réduits à leur plus simple expression: le vaudeville, le burlesque se transforment en danse mortuaire. Chez Beckett, on marche, on se déplace, on va et on vient jusqu'à la conclusion logique de ce périple: l'immobilité (l'asphaltisation ultime?) qui, au lieu qu'on attende Dieu, fait qu'on se résigne à vivre dans la mort ${ }^{8}$.

C'est toute une tradition que je pourrais mettre au jour en tirant le fil des dialogues, des emprunts, des filiations; je pourrais parler d'Auster et de sa bien connue trilogie newyorkaise, dans la mesure où les enquêtes conduites par ses divers protagonistes buttent sur cette immobilité de sujets qui, malgré leurs affabulations cosmétiques et identitaires, sont réduits à devenir des témoins du néant. Je pourrais en revenir à L'homme qui tombe (The Falling Man, 2007) de Don DeLillo, où les personnages, qui habitent dans des univers de conventions, constatent leur nullité, leur absence de profondeur à la suite de l'attentat terroriste du 11 septembre 2001.

Tout cela me ramènerait en fait au début du texte de Widmer, lorsqu'il prend pour exemple l'œuvre immense de Charlie Chaplin. Cela fait retour au segment consacré aux temps modernes, à la description d'une chaîne de montage en proie à la dislocation, à la figure pervertie d'un rapport authentique au monde et à l'enchaînement des idées et des actes. Ce n'est pas tant l'extrusion, ou le refus d'être extrudé qui serait à réfléchir, mais bien la dislocation parce que cette dernière se pense en termes de perte.

Widmer a cherché au plus intime de ce qu'est la rébellion, tentant de cerner comment se manifeste cet acte de défiance à l'égard de l'autorité. J'ai failli écrire un «acte de défense» au lieu de «défiance»: écrire noir sur blanc ce lapsus, ce n'est pas seulement ici faire l'aveu d'une mauvaise prononciation, la langue qui fourche, deux voyelles qui sont confondues. Ce lapsus est la manifestation langagière d'une explosion du sens, d'un terrorisme qui cantonne l'individu dans son claustrum, son petit bunker identitaire qui lui permet de survivre tout en redoutant son semblable. 
La rébellion, comme la révolte chez Camus, la revendication de la personnalité enfin libérée de ses chaînes chez un Emmanuel Mounier, de l'existentialisme chez un Sartre, traduisent l'idée d'une opposition à un monde qui existe hors de soi, qui peut faire l'objet d'une défiance. Cette dernière expression revient à quelques reprises dans l'œuvre de Widmer, en l'occurrence The Literary Rebel, afin de circonscrire l'éthos de sujets qui refusent l'ordre établi:

\begin{abstract}
Rebels must also reply to those in power that they "refuse history", that their choices of moral libertarianism and idiosyncratic art and social directness and intellectual iconoclasm were not aimed at practical power, whatever the circumstances. The point seems incomprehensible to many of those, whether presidents or clerks, who feel themselves wired up to the manifest forces of their times. On the one hand, those rebels who turn to power and success and conformity with overt history do so only by negating the rebellion. On the other, the consistent rebel must even mock rebellion and truth - his appropriate claim to esoteric power; many do, with an ultimate (though hardly maintainable) nihilism which provides the rebels' unique terror, and strength. Paradoxically, the rebel cannot have direct power, or he ceases to be a rebel; yet he must have some significant relation to, or effect on, power, not only to avoid futility and capriciousness but to define himself against (WIDMER, 1965, p. 30-31).
\end{abstract}

Être rebelle, cela veut dire que l'on met en cause, avec une violence peu commune, la société à laquelle on appartient. Cette défiance ne connaît pas de limites; c'est un parler contre les institutions et les gouvernements, le pouvoir de l'argent, la description du conformisme.

Dans le contexte des années 1950-60, riche en rébellions, contestations, renversements contre-culturels de toutes sortes, la condition de rebelle ne reposait pas uniquement sur un statut social, une marginalité, l'adoption d'un point de vue excentrique, en tous les cas détaché des servitudes de la société. C'est le témoignage d'une volonté opiniâtre. Au-devant des coups, des attitudes réfractaires, le rebelle conteste le pouvoir de l'autorité. Comme bien des témoignages de cette époque d'après-guerre, le discours de Widmer misait sur le pouvoir du langage, seule manière d'énoncer une vindicte, une colère.

Aux États-Unis, il y a eu le mouvement pour les droits civiques, la marche sur Washington et, plus tôt, l'occupation de l'université de Stanford, les nombreuses manifestations sur les campus des universités de l'État de la Californie. C'est la 
guerre du Viêt Nam qui se manifestait dans toute sa violence. L'Amérique était en pleine effervescence. Le sursaut européen que représenta mai 1968, deux ans après la réédition de The Literary Rebel, peut être perçu comme un mouvement retardataire, la re-symbolisation, sur fond de lutte politique prolétaire et marxiste, adaptée au monde étudiant, de ce qui se joua cinq ans plus tôt sur la Côte Est et la Côte Ouest des États-Unis.

Tandis que Norman Mailer connaît le succès avec Les nus et les morts (The Naked and the Dead, 1948), que V.S. Naipaul quitte Port of Spain pour Londres, les intellectuels français de l'immédiat après-guerre Albert Camus, Jean-Paul Sartre et Simone de Beauvoir ont de toute évidence le sentiment que l'histoire joue pour eux, que l'avenir du monde leur appartient. Toute une génération dans la mouvance de la contreculture s'est par ailleurs énamourée de la certitude d'être au monde au bon moment. Être là au bon moment, cela voulait dire être là quand la rébellion fait rage?

À l'instar de Diogène le plus célèbre des cyniques, le rebelle qui se moque en toute impunité de la société dans laquelle il vit peut avoir une attitude qui prend des airs de prétention narcissique et vire rapidement à la complaisance, à l'autosatisfaction. Son propos perd alors en cohérence, et Widmer se plaît à le signaler. Cette énonciation rappellerait les plaisirs adolescents de la révolte contre l'autorité.

Une autre question se pose aussi : qu'en est-il lorsque cette autorité est diffuse, qu'elle ne peut plus être ramenée à un certain nombre de symboles (l'argent, le pouvoir, l'autorité) qui incarneraient la rectitude de la vie des bourgeois? En effet, cette opposition en apparence tranchée du bourgeois et du bohémien, du hobo et du propriétaire, du vagabond et du salarié qui reçoit son salaire à chaque quinzaine, ne tient plus, dans le monde actuel pour qui la rébellion est devenue au mieux une expression confuse, une revendication que les déclassés, pauvres, chômeurs, itinérants, n'osent même plus employer. En d'autres termes, la rébellion serait un discours d'antiquaire, serait du réchauffé.

Chez Widmer, c'est le soi qui est mis en relief, le recours à l'insulte, à la violence, à la bravade, ce qui est d'une grande importance tant le rebelle essaie, de manière systématique, de parader, de démontrer avec force gestes combien il s'oppose à l'ordre établi. Ainsi dans sa description de l'œuvre de Mailer Widmer parle-t-il de téléologie salvatrice qui correspondrait à un monde cosmique, voire à l'Inconscient: 
As in most mystical patterns, Mailer's purging or destruction of the ordinary self and consciousness - by excremental insults, violence, orgy, demonism, frenzy and bravado - rests on the salvational teleology that the above or the below, the cosmos or the unconscious, will salvage all. Under the terrorist, Rousseau; above the immoralist, transcendental mysteries. Short of the mystery itself, all should be «creative rage». At the individual level, apparently, that means «acting out»; at the literary level it means, from the evidence, ornately frenzied art. Thus, in our amorphous rationalized society, the rebel asserts himself as a religiously sanctioned exemption from organized blandness and technological decorum (WIDMER, 1965, p. 184).

Le rebelle ne cesserait donc de combattre une autre version de lui-même, la représentation d'un narcissisme dédoublé, sous la forme d'un idéal du Moi à la fois négatif et positif.

Ceci étant dit, cette perspicacité de la part d'un écrivain-essayiste qui cisèle son œuvre ne doit pas cacher la dureté qu'elle contient, à savoir de ne pas faire de quartier, de ne pas hésiter à couper un doigt ou deux, à faire mal à son lecteur, à assassiner un Jack Kerouac ou un William Burroughs. Qu'aurait-il pensé de Richard? Le pire sans doute. Il aurait fustigé l'enhardissement extrême contenu dans son Journal, aurait peutêtre été scandalisé par l'hermaphrodisme du héros, aurait trouvé à redire sur la qualité de l'écriture... Richard aurait certainement rejoint les cibles de la prose enragée de ce touche-à-tout qu'est l'auteur de The Literary Rebel, dont certains passages sont d'une clarté foudroyante - aux côtés de quelques bavardages, il est vrai.

En 1964, Dylan chantait «The times are a-changin'», sorte d'hymne de l'époque pour une jeunesse qui croyait que tout allait effectivement changer, qu'il fallait s'adapter, savoir nager sous peine de couler comme une pierre... «Come writers and critics / Who prophesize with your pen, / And keep your eyes wide / The chance won't come again» chantait Dylan au deuxième couplet de sa chanson. Il en appelait aux écrivains et aux critiques, aux politiques, aux parents, les mettant en garde contre le changement à venir... Mais voilà, cinquante ans plus tard, rien n'a vraiment changé. À l'âge où nous sommes en fait, celui de la défiance extrême, la tartufferie esthétique est un acte d'autant plus 
véhément qu'elle ne s'accompagne d'aucune connaissance, que la loi ne s'embarrasse pas de justice, qu'elle incarne, pour elle seule, le point de départ et la destinée d'une compréhension du monde irrémédiablement faussée. Rien n'aurait donc changé? Penseurs et artistes de la bohème d'aujourd'hui seraient les mêmes qu'hier, malgré un changement de vocabulaire, les années contre-culturelles ayant laissé place à l'académisme de l'espace public? Seraient-ils de nouveau face aux mêmes impasses, difficultés d'énonciation, apories qui prévalaient dans le contexte, certes plus libertaire, des années 1960?

Il ne s'agit pas de parler de l'aliénation du créateur au cœur d'un monde qui ne le respecte pas, mais de la sophistication pathétique de la création sous la forme d'une thérapie individuelle, d'un art auto-créé tenant lieu de loisir, de défense contre l'ennui, de commercialisation de la vie intellectuelle. La création serait devenue pire qu'un spectacle; elle serait une forme d'hyperspécialisation qui serait le lot des élites. Et Widmer le dit avec une méchanceté sans merci:

The pathetic sophistication of art as personal and social therapy, as hobby and time-off defense against boredom, as commercial entertainment and production, as academic and intellectual specialization, as polite resentment and covert superiority - in short, «creativity» as the American addenda to bovarysme - relates neither to significant art nor to genuine rebellion. Surrogate culture and defiance surely serve practical purposes, and some imperatives of those in power: they inhibit and falsify deeper and fuller discoveries of discontent. The millions of Americans who practice, or vicariously partake of, self-expression and nonconformity only in the arts have found a convenient way of not doing them in their institutions and the streets. The categories reveal the trick: « art» but not ordinary work is «creative». «Imaginative writing» is something to take a course in, not something to do in public statements, letters, and all that dark wood of printed matter constantly growing around us. Pictures and poems, but not our cities and people, reveal «form» and «feeling». «Style» is proper in art, irrelevant in life (WIDMER, 1965, p. 146-147).

Widmer s'en prend à l'idiotie bohémienne de ses contemporains, de la jeunesse enchanteresse qui, par monts et par vaux, sur la terre américaine, prétend créer de nouvelles utopies. Que nous dit-il, en effet, si ce n'est que la valorisation de la créativité dans les années 1950-1960 s'apparenterait à un loisir, à un acte sans grande conséquence? 
Que nous sommes bien loin du souhait d'une vie meilleure, partagé par les situationnistes, les beats, les rovers et les ramblers américains?

À y regarder de près, la contestation du bourgeois, puis des représentants de la classe moyenne, c'était, dans les années d'après-guerre, une attitude qu'il était possible d'adopter. Ce bourgeois d'ailleurs avait bien changé. Si l'on pense à la France d'avant la révolution de 1848, c'était l'homme de lettres qui possédait de grandes quantités de livres dans sa bibliothèque privée, d'œuvres d'art dans son musée particulier, bref qui détenait une richesse culturelle inouïe en plus de ses capitaux. Avec la révolution de 1848, cet homme-là avait laissé place en France, aux commerçants, aux négociateurs, aux personnages représentant ces capitaines d'industrie qui, par la suite, allaient redéfinir le temps présent. C'est ce bourgeois qui était vivement critiqué, entre autres, par les bohémiens artistes qui étaient bien souvent eux-mêmes fils de bourgeois, héritiers d'une culture lettrée qu'il faut contester.

Or, lorsque Widmer publie en 1965 sa diatribe, le monde bohémien avait connu ses heures de gloire. L'esprit bohémien, avec son dilettantisme, sa manière d'être tout en refusant pour un temps l'héritage familial, sa façon de s'en remettre au monde de la rue qui semble plus vrai, qui possèderait une acidité et une nouveauté aux antipodes de la transmission d'un savoir parental était maintenant condamné, dans le contexte des années d'après-guerre, à céder de son pouvoir, de son autorité à une classe moyenne en apparence illettrée qui tentait de se mettre au goût du jour.

La mode de l'univers bohémien, en tant que succédané des années beat qui connurent leur apothéose dès la fin de la Seconde guerre mondiale, s'avère au bout du compte, selon Widmer, un regard conformiste sur le monde, la description de bourgeois apeurés par le spectacle d'une violence... qu'il importe de canaliser autrement. L'univers bohémien est ce bovarysme, ce provincialisme des petits notables de province qui, s'emparant de l'intensité et de la flamme des désespérés, avec l'appétit des constructeurs qui créent des monticules de pensées, usurpent la rébellion, la solitude, en font la matière d'un lieu commun. C'est en cela que l'on peut parler de dislocation.

$\mathrm{Si}$, avec la conscience de faire ce mouvement très rapidement, je trace le passage de l'univers bohémien à sa représentation stéréotypée dans l'univers culturel des années 
1960, c'est que le propos de Widmer, enraciné dans le monde états-unien, traduit bien ce passage obligé, cette re-symbolisation stéréotypée du monde de la marge, de la contestation, de la transgression. Ledit propos est lui-même la reprise ironique du discours qui, en Europe, dès le 19e siècle, canonise en quelque sorte l'idéal bohémien, seule possibilité pour se sentir poète, écrivain, homme de lettres dans un monde en proie à l'atonie de la révolution industrielle.

Mais décidément les temps ne changent guère... On ne saurait contester l'analyse de The Literary Rebel, pas plus qu'on ne saurait en désavouer la cruelle actualité, plus de cinquante ans après sa publication, tant la portée industrielle de la créativité artistique est aujourd'hui à l'agenda de tous les décideurs publics à l'échelle planétaire.

Widmer, avec les références qui étaient les siennes, percevait le bovarysme comme la forme suprême d'un ennui; nous dirions aujourd'hui que ce n'est pas tant l'ennui et l'asthénie dépressive qui s'ensuit qui caractérisent l'époque, mais la métamorphose de la créativité sous l'aspect d'une fluidité commerciale. Il s'agit d'un nouvel art de faire industriel qui, avec les moyens du bord, régente les aspects de la vie commune. Il convient donc de rester sur ses gardes, de ne pas céder à l'euphorie ${ }^{10}$, car l'éloge du nomadisme bohémien, s'il est séduisant, n'en demeure pas moins aujourd'hui un discours à forte connotation touristique, même dans le contexte des politiques culturelles renaissantes des métropoles, notamment au Canada (je pense précisément à Toronto et à Montréal).

Ainsi, l'identité bohémienne serait à saisir, sous son aspect promotionnel, comme la description d'un monde dont la créativité a déjà été extirpée de la manière la plus violente. Greenwich Village est définitivement devenu un lieu de touristes lorsque les derniers artistes, ne pouvant plus payer leur loyer, ont migré vers des cieux plus cléments. Dans un autre contexte, Richard Lloyd écrit dans son Neo-bohemia Art and Commerce of the Post-industrial City (2010) que le quartier Wicker Park City, à Chicago, perdit définitivement son âme lorsque des chaînes de production de téléréalité firent leur apparition dans cet espace culturel non-gentrifié. Ainsi, l'émission The Real World a proposé à ses téléspectateurs, lors de sa onzième saison, en 2002, un épisode sur le thème 
«Devenez un artiste aujourd'hui dans le quartier le plus hip de Chicago» (LLOYD, 2010, p. 147-153). Or, on ne peut créer un quartier de toutes pièces, la culture n'est pas un phénomène spontané, auto-créé. En effet, la culture ne se transmet pas rapidement, il faut du temps, il faut de la latence, de la résilience et aussi des conflits.

Mais voilà, la créativité est à l'agenda des discours des élites. Loisir, expression personnelle, redécouverte de soi, approfondissement de l'introspection, croissance personnelle, tous ces discours décrits dans le contexte des années 1960 ont aujourd'hui plus que jamais la cote. Si le monde contre-culturel d'hier, héritier de l'univers des beats, justifiait cette créativité au nom d'un ressourcement individuel, il faut croire que la bonne vieille idéologie de la subjectivité, pleinement affirmée, a un pouvoir de séduction extraordinaire encore aujourd'hui. La lecture du Journal d'un hobo de Richard nous a aidés à comprendre cela. En fait, l'époque présente se caractérise par la mise en scène de ces identités fluides, mobiles, créatives, résilientes, qui sont l'exact contrecoup d'une perception traumatique de l'univers. Joie et détresse alternent. Rires et pleurs composent des sarabandes étonnantes. Tout se passe comme si la bipolarité, à l'heure des troubles identitaires de l'humeur, cédait la place à une nuée de mots, de pensées diverses, de bribes poétiques et de jointures sémantiques. Nous sommes l'un et l'autre, à la manière du narrateur-protagoniste du Journal d'un hobo. Nous ne connaissons plus les heurts de l'identité, l'obligation de choisir, de savoir se situer dans le monde.

La créativité libertaire s'inscrit au programme des décideurs et des gouvernants qui prétendent avoir raison, puisqu'ils sont eux-mêmes les bébés-boomers tardifs qui, au cours des années 1970, ont vécu cette révolution culturelle de la prise de conscience libératrice. Les cartes sont dorénavant brouillées, le fantasme du hobo-bohémien récupéré, voire instrumentalisé. Il est devenu une chimère sur laquelle on fonde une vision politique très éloignée de ce qu'auraient souhaité les bohémiens parisiens de la fin du $19^{\mathrm{e}}$ siècle et les hobos du $20^{\mathrm{e}}$ siècle. Le $21^{\mathrm{e}}$ siècle nous fait clairement voir autre chose. Les anciens soixante-huitards sont devenus des sous-ministres, des ministres, dans différents appareils gouvernementaux. Au Québec, le discours nationaliste, représenté par le Parti québécois, est à la recherche, pour une ixième fois, d'une voie de sortie qui permettrait de repenser cette énergie libératrice qui s'était affichée lors de l'élection de ce parti en 1976. Après 
deux référendums, en attendant le troisième, quelque chose se répète, se délite, comme si la créativité «populaire», cette idée que le peuple est en marche, et qu'il rencontrerait son destin, avait laissé place à l'atonie dépressive. Tout se passe comme si la croyance en une créativité émancipatrice, à l'âge de la prise de parole, des revendications réparatrices d'un sujet collectif exploité (des populations homosexuelles aux Premières nations), avait laissé place à un consumérisme de la créativité, sa mise en marché. L'idéal d'hier semble être devenu un stéréotype.

Qui se soucie vraiment aujourd'hui, dans un monde soumis à la récréation, de faire œuvre de créativité, à l'heure des grandes coupes budgétaires imposées par un gouvernement ayant décidé de mettre la culture à terre, de tuer dans l'œuf toute possibilité de création, et sans doute pense par là tuer toute contestation possible? Trouvera-t-on que je vais trop loin, et entendra-t-on le cri derrière la plainte face aux appareils politiques qui se sont emparés de la créativité pour la soumettre à une récréation lucrative?

Cela, Widmer ne pouvait en cerner toute l'ampleur au cours des années 1960. Il ne pouvait pas savoir que dans le contexte californien où il créa son œuvre, un Jerry Brown, plus jeune gouverneur de l'État de la Californie, mettait déjà en relief cet essor créatif qui donnera naissance à la fameuse Silicon Valley. Peut-être est-il réducteur d'opérer une relation de consécution entre l'imaginaire californien des années 1960 et la mise en marche d'un appareil politico-industriel créatif. Il n'en reste pas moins que l'esprit contre-culturel des années 1960 a connu des métamorphoses de grande ampleur. Il a permis l'émergence puis la consolidation, à l'échelle mondiale, du pouvoir créatif, tel qu'exprimé, entre autres exemples, par le statut incontestable de la Silicon Valley dans le domaine des technologies de l'information et de la communication.

Mais ces grands mouvements de capitaux, ces mutations de l'appareil industriel, ces apparents changements de paradigme quant à la manière dont nous percevons et disons le monde qui nous entoure, tout cela ne saurait masquer le fait que la «créativité néolibérale» est de peu d'utilité lorsque nous sommes épuisés, exsangues, vidés d'une énergie sans laquelle nous ne pouvons répondre à l'attaque, à la mise en cause, à l'expression agressive dont l'autre est, plus souvent qu'autrement, l'énonciateur.

La créativité, cette fantastique redécouverte de soi, a toutes les apparences d'une 
énonciation narcissique. Elle ne possède plus l'ingrédient nécessaire pour prendre son envol. Il faut tenir compte de cette fatigue, lorsqu'il s'agit de prendre au sérieux ce que la détresse consumériste de la vie quotidienne, à l'ère de l'hyper-modernité, impose de sacrifices, de violence que l'on commet contre soi, pour posséder un bien, en devenir propriétaire, ou encore, plus simplement, en obtenir le droit de location de manière à ce que nous soyons, tous ensemble, rassemblés dans cette parade commerciale des propriétaires en sursis.

Widmer se bat donc contre l'idiotie bohème et nous sert à mieux lire la créativité libertaire qui est notre lot. Mais justement, qui a lu Widmer? À première vue, l'auteur est méconnu, sans que l'on sache trop pourquoi. À bien y regarder, on se pose des questions : est-ce par la faute de son appétit de cruauté, d'une attitude à la fois éclectique et rageuse, que cet auteur est demeuré à l'écart? Est-ce que c'est parce qu'il n'appartenait pas au monde universitaire, à ses institutions, aux lieux reconnus qui font de la transmission du savoir - moins une véritable épreuve qu'un rite de passage, dans bien des cas une cooptation d'ailleurs? Est-ce que les universitaires le redoutent, car sa méchanceté n'épargne pas leur milieu? Il a en effet la dent dure:

Yet we also do not have an « intelligentsia » of any proportions which stands outside the official order as a rebellious group or class. Our mass-technological-bureaucratic societies reveal shrewd self-preservative instincts; other societies threw intellectuals into the streets where they became troublemakers. Our way is to give the literate and intellectual great opportunities to forego those traits and take up, in proper disguise, commercial and technical roles. Or they can keep a modicum of their devotion to the life of letters and arguments and become specialists, properly institutionalized and insulated, practicing their expertise. The contemporary effulgence of literary criticism becomes one such form of institutionalization, apparently providing a specialist refuge for moralists and theologians and philosophers and artists as well as rebels. Replete with some of the appropriate learning, techniques and issues of professional literary study, quite an odd variety of intellectual passions find an outlet, however limiting in «criticism» (WIDMER, 1965, p. 160). 
Quel propos dévastateur! Widmer était à sa manière un anarchiste et un fort en gueule, il ne faisait pas partie de la coterie littéraire de son époque. S'en prendre à Norman Mailer ou à Paul Goodman avec une virulence peu commune, c'était accepter de lutter contre des Géants. Widmer était sans doute un observateur qui, parce qu'il pouvait se maintenir à distance du monde qu'il écrivait, était en mesure d'exprimer un cynisme, une virulence qui lui ferma bien des portes. Il dit de manière extraordinairement limpide que la spécialisation, l'obéissance à un ordre professionnel implicite (à savoir celui des gens de lettres) a des conséquences néfastes. D’abord, cette hyperspécialisation forme des sujets convaincus de leur importance dans le domaine culturel, parce qu'ils sont en quelque sorte prédéterminés par des habitus au sens où l'entendait Pierre Bourdieu à propos des phénomènes sociologiques de distinction de classe. Ensuite, cette même hyperspécialisation est une manière de morceler le monde du langage, de la culture, de pratiquer les formes de désolidarisation de l'univers intellectuel, comme si chacun traitait, sans avoir le moindre intérêt pour ce qui va au-delà de sa pratique immédiate, des microstructures, des phénomènes singuliers et localisés, des vignettes historiques limitées aux périodes les plus brèves, comme s'il fallait, avec le souci d'un entomologiste, percer, dans l'abstraction la plus forcenée, la racine de notre identité profonde.

Ce silence qui entoure l'œuvre est d'autant plus troublant que ce qu'elle nous dit à propos des années 1960, à savoir la refonte de l'esprit bohème dans une fantasmatique qui concerne une certaine élite, pourrait sans aucune difficulté s'appliquer en presque totalité à notre époque. Là est le mérite de l'auteur: nous faire réfléchir à notre propre situation. Il faut prendre au sérieux son avertissement qui, malgré son lot d'exagérations, explique que l'art de la rébellion est devenu un sophisme dès les années 1960.

Depuis, quelque chose s'est accéléré, puissamment, comme si le métronome, cet outil de notation du rythme, s'était brusquement déréglé. Arythmie, tension cardiaque, douleur dans tous les os du corps, maux de tête et migraines, brutales décompensations somatiques qui se manifestent sans avertissement, tel est l'ordinaire des travailleurs de l'infâme, ces nouveaux sujets qui ne sont plus des prolétaires, mais des travailleurs autonomes pour lesquels l'injonction de la banalité, cette forme suprême de l'ennui dont Widmer dit qu'elle caractérise les classes moyennes au sortir de l'après-guerre, n'est plus de mise. 
Nous avons l'habitude de concevoir le regard comme une façon d'être dans le monde, une perception qui fait intervenir, sous les formes du proche ou du lointain, la possibilité d'être aux aguets, ce qui signifie l'acte de sur-veiller, de s'assurer que nulle menace ne vient contrevenir à notre sentiment de liberté. Qui est aux aguets veille, sur-veille, se met dans une position de vigilance et fait de la perception une forme d'auto-défense.

Peut-on imaginer, par ailleurs, l'apparition de brèches au cœur du réel, de zébrures et de failles qui seraient le signe que les territoires que nous habitons peu à peu perdent de leur consistance assurée, cessent de représenter des sites instaurateurs d'empires et de forteresses? L'empreinte autoritaire du pouvoir se fait visible partout - n'est-ce pas ce que met en scène un auteur comme Antoine Volodine, de manière criante? Pour qui n'est pas encore paranoïaque, il suffit de marcher dans les villes d'Occident, de constater à quel point nous sommes surveillés de toutes parts, numérisés, catalogués, répertoriés, catégorisés, pour que la paranoïa réelle, celle qui est induite par la violence de l'empire, s'empare de nous. Ce propos est peut-être extrême, mais il est là pour en revenir à la question puissante et jamais épuisée de la limite. Le hobo, qu'on se le dise, se définit par la limite.

Le marginal, c'est celui qui est dans la marge, qui marche dans les rues sans y être asphaltisé et que des générations de poètes ont nommé le vagabond, le clochard, le clodo, le hobo. Il n'arrête pas son regard sur un objet précis, avec des mouvements mesurés du corps et d'infinitésimaux battements des paupières, son regard balaie l'univers de la rue, du trottoir, du sol, de la souillure du sol, des objets qui jonchent le sol. Il ne cesse de faire des arrêts, des mises au point. Parler de la limite, c'est donc prendre en compte ce qui n'en a pas, ce qui est repoussé au-delà : le hobo ne fait pas autre chose, le bohémien, lui, en joue.

Le bohémien s'est dévoyé avec le temps, constate Widmer comme d'autres le feraient aujourd'hui en quelques clics sur Internet, en cherchant ce qu'est «le style» vestimentaire hobo, reléguant aussi cette posture au rang de jeu comme on a pu le faire avec la mode hippie. Ainsi est-il consternant de constater les pages consacrées à ce «style» qui permet d'être «tendance», un comble, n'est-ce pas? Pour faire «hobo», une femme doit avoir le sac qui convient. Sur les sites concernés, les sacs «hobo» sont identifiés avec des marques plus ou moins connues, comme Chloé, Marc By Marc Jacobs, Diane de 
Furstenberg ou encore Michael Kors - les prix ne sont par contre pas «hoboïsés», car si vous voulez avoir le «style hobo» il faut débourser dans les 560 dollars l'article et plus de 1300 pour le plus cher (Heritage Boston) ${ }^{11}$. Mais le sac ne suffit pas pour vous transformer en «hobo»: d'autres sites mettent l'accent sur le look total ${ }^{12}$ ou sur des variantes assez paradoxales, voire oxymoriques telles que «hobo haute couture» (John Galliano), «hobo chic» voire «hobo geishas» ${ }^{13}$.

Cela ne s'arrête pas là, il y a, plus étonnant encore, l'association entre deux marques, Cinelli et Moleskine, pour prendre la route à la manière d'un... cycliste hobo: «Comme lorsqu'ils voyageaient autrefois à bord de cargaisons, l'esprit du hobo vit chez le voyageur moderne qui enfourche son vélo à la recherche de possibilités inconnues ${ }^{14} \gg$. Le jeu est affirmé, poussé même à l'extrême, et l'on peut dans une certaine mesure se poser la question du bon goût, puisque le carnet hobo, là pour noter ses impressions lors de balade, est décoré avec des icônes Hobo - ces fameuses icônes que les hobos se laissaient entre eux, codes à leur seul usage pour indiquer danger ou abri, point d'eau aussi - et contient les règles et codes du cycliste hobo! C'est même dit clairement: «Jouez avec les icônes Hobo pour laisser des messages codés sur la route grâce aux autocollants inclus 15 ». Les hobos ne seraient-ils donc plus que des être folkloriques qu'ils conviendrait d'actualiser comme c'est le cas du «hobo fashion»?

À regarder ici et là sur Internet, alors même qu'on vit aujourd'hui une crise économique, l'univers des hobos est souvent restreint à un discours folklorique que l'on veut sympathique. À ce titre, en contrepartie de ces bobos qui jouent un court temps les hobos, qui se déguisent avant de reprendre leur costume de ville, les hipsters à vélos représentent ce mouvement caricatural. Comme toutes les modes fondées sur les règles du revival et du vintage, il faut tenter de renouer avec une Amérique qui n'existe plus depuis longtemps. À la suite du Beat recyclé en icône de l'Americana sous l'influence du Hipsterism, la mise en scène du hobo deviendrait l'expression d'une nouvelle quête d'authenticité dans le meilleur des cas, d'un jeu dans le pire. Cette rébellion, à regarder la mode des hobos et des hipsters, s'est donc tue, ou du moins elle sonne faux trop souvent.

Je ressens une profonde aversion à l'égard de ces modes qui n'ont aucune singularité et sont des copies conformes d'un passé que tous souhaitent idéaliser. Untel porte le fameux 
Pork pie hat sans savoir qu'il s'agit d'une pièce musicale de Mingus inspirée du chapeau que portait le saxophoniste Lester Young. Combien de jeunes adultes sont affublés de ces barbes touffues qui témoignent, ainsi que l'annonçait Barthes, d'une connotation dont les origines, comme toute mythologie, est diffuse? Mon aversion est certainement l'aveu d'une quête de vérité, comme s'il ne fallait pas dégrader une icône, que ce soit la barbe d'Allen Ginsberg ou le chapeau (de fourrure de mouton de Perse?) que porte Lawrence Ferlinghetti en 1975 lors la fameuse photographie prise au Café Trieste.

Mais qui suis-je pour me transformer en gardien de la tradition? Je n'étais pas né quand le mouvement Beat fit son apparition. Je suis au mieux un enfant de la contreculture dont je n'ai connu que l'aspect dénaturé. Quel aveu un peu ridicule, je m'en rends compte! Ne suis-je pas l'un des héritiers perméables à ce que représente, pour une génération, une classe sociale, un mouvement culturel d'envergure, la tradition? À suivre ce point de vue, il y aurait donc, pour chacun d'entre nous, une méfiance à l'égard de la nouveauté, par définition dérangeante. Alors que chacun vieillit, la tradition prendrait la forme d'un paysage culturel familier, un univers dans lequel on se trouve somme toute à l'aise, entouré de points de repère.

Pourtant, les figures du hobo ne me sont pas familières. Si je me rappelle mon enfance dans une des banlieues du Nord de Montréal, je me souviens (mais ne s'agit-il pas, comme le disent les psychanalystes, d'un souvenir-écran?) d'hommes juchés sur une plateforme. Dans mes songes à mi-chemin entre le sommeil et l'acuité du jour (car je me demande si j'ai rêvé tout cela par dépit, ou si je l'ai réellement vécu), je vois ces hommes assis sur ce qui semble un échafaudage de métal rouillé, alors qu'ils tentent de se protéger du soleil. Sont-ce des hobos? C'est à ce moment, je le sais, que la fiction prend le pas. Cette fiction qui se met en place concerne mon enfance, et déjà elle permet en quelques traits de voir comment une fiction commence à se dessiner à partir de très peu, quelques souvenirs épars dont on ne sait pas au bout du compte qu'ils sont vraiment des souvenirs.

Comme tous les autres, j’ai construit ma mythologie. L'expression est démesurée, elle témoigne sans doute d'une confiance en soi que mon propos met pourtant en doute. Qui a soif de mythologie personnelle veut certes se rehausser, mais l'exercice ne coïncide pas toujours avec une fabulation qui peut verser dans la mythomanie. Dans la façon 
dont chacun forme ce qui deviendra son héritage culturel, il y a un immense besoin de reconnaissance, comme s'il fallait adhérer à l'époque, se faire une place, être (re)connu. Se construire une mythologie, c'est aussi s'enhardir.

C'est dans cet état d'esprit que d'autres que moi essaient de pratiquer l'art du braconnage, de mettre en cause l'autorité, loin des fêtes foraines, du cirque culturel dont l'artiste, nouveau desperado, incarnerait, au plus offrant, le spectacle d'une lutte infinie cantonnée au registre de la tartufferie esthétique. D’autres voix aussi se font entendre, dans l'héritage non pas glorieux, mais cette fois terrifiant d'un hobo affamé dans un monde sinistré, sans foi ni loi, un monde grevé par la violence. Autrement dit, ces voix s'entendent au cours d'une marche initiatique.

Dans les années 1960, il y a eu une grande élaboration dans la construction fantasmatique de la figure du hobo et en même temps le texte de Kerouac, A Vanishing American Hobo (1960), sert de transition, de marque qui désigne la fin d'une réalité, mais certainement pas la fin du fantasme du hobo, dans sa connexion avec la liberté et la contestation - quelle qu'en soit sa modulation, sa prise de position, pour avec Kerouac, contre avec Widmer. Et puis, la figure s'effrite en même temps que se multiplient les œuvres dystopiques qui nous racontent inlassablement la fin du monde, d'un monde, le nôtre. Or, ces œuvres, comme les précédentes, s’appuient sur le mythe américain de la frontière et de la loi.

Les discours freudiens faisant l'objet d'une réévaluation lacanienne mettaient l'accent sur le principe de la loi du nom du père, qui trouvait son efficacité dans le cadre d'une analyse des processus psychotiques et paranoïdes. Cette mise en scène du père s'apparentait à une mise en cause d'une autorité dont nous aurions, en apparence, bien de la difficulté aujourd'hui à trouver des équivalents. N'est-il pas fréquent de dire que l'univers délocalisé dans lequel nous habitons, conséquence d'un postmodernisme économique et financier, a entraîné des modifications quant à nos représentations de l'autorité? Cette dernière n'aurait plus de caractère unificateur. Elle serait multipolaire, ce qui veut dire que la loi du nom du père aurait éclaté en fragments, que la socialité (la gouvernance, les 
transactions entre citoyens, le principe de la loi souveraine ramenée au bon jugement du père de famille) serait dans l'obligation de se redéfinir. Peut-être.

Mais qu'en est-il dans la fiction? Quand il s'agit de reprendre, de réinventer une figure fantasmatique comme le hobo qu'on ne peut en aucun réduire, on l'a vu, au principe de révolte, de contestation, que lui a donné en héritage le bohémien-artiste français de la fin du $19^{\mathrm{e}}$ siècle?

Traverser le monde connu pour trouver sa propre identité au bout du compte, c'est d'ailleurs le point de vue partagé entre Richard, Kerouac, dans une version positive, et McCarthy, auteur contemporain, dans une veine dystopique quand l'enhardissement se heurte à la loi du père. Mais au fait, qu'advient-il quand il s'agit d'examiner quelques œuvres américaines d'aujourd'hui qui reprennent en leur cœur la problématique de la loi du père? Avec ces questions au bout des lèvres, je souhaite à présent parler des affres de la loi et de la justice, sans doute parce que cet adoubement fait en sorte que la loi, pour l'écrivain, ne peut être une justice égale pour tous, dans la mesure où il ne connaît qu'une seule voix, la sienne.

\section{Références}

ANDERSON, Nels. In. SINGLY, François de (Org.). Le Hobo sociologie du sans-abri. Trad. par Annie Brigant. Paris : Armand Colin, 2011.

CLERVAL, Anne. Les bobos. Critique d'un faux concept. Cybergeo: European Journal of Geography [En ligne], Revue de livres, mis en ligne le 17 mars 2005, consulté le 10 mars 2017. URL: http://cybergeo.revues.org/766.

CÔTÉ, Jean-François. Littérature des frontières et frontières de la littérature: de quelques dépassements qui sont aussi des retours. Recherches sociographiques, Vol. 44, № 3 , septembre, décembre, 2003, p. 499-523. URL : http://dx.doi.org/10.7202/008204ar

FERRON, Jacques. La nuit (texte de 1965, présenté par Diane Potvin). Paris: Fernand Nathan Éditions / France Québec, 1979, coll. Classiques du monde.

LLOYD, Richard. Neo-bohemia: Art and Commerce in the Post-industrial City, [2006] 2nd ed. New York and London: Routledge, 2010.

RICHARD, Jean-Jules. Le journal d'un hobo. L'air est bon à manger. Montréal: Parti pris, 1965, coll. Paroles 6.

WAGNIART, Jean-François. Le poète, le réfractaire et le vagabond. In: Le vagabond à la fin du 19e siècle. Paris, Belin, 1999. 


\section{WIDMER, Kingsley. The Literary Rebel, Carbondale, Illinois: Southern Illinois University Presse, 1965.}

\section{Notes}

1 Université de Montréal, Quebec, Canada. simon.harel@umontreal.ca

${ }^{2}$ C'est ainsi que la représentation de l'idiotie, au même titre que celle de l'étranger, de l'exclu de la société, du marginal, qu'il s'agisse de l'original, de l'iconoclaste, de l'excentrique, représente à peu près les mêmes balises, les mêmes points de capiton dès lors qu'il s'agit de fonder une argumentation.

3 Tempêtes de poussières qui se sont abattues dans la région des Grandes Plaines aux États-Unis et au Canada dans les années 1930.

${ }^{4}$ Il s'agit d'une part d'Henry Murger, Scènes de la vie de bohème (1888) que Puccini adaptera pour l'opéra et d'autre part de Colin Wilson, The Outsider, 1956. On pensera aussi au texte de V.S. Pritchett qui a pour titre La vie de bohème (1943).

5 Voir: (CÔTÉ, 2003).

6 Nels Anderson consacre une partie de son étude à ces créations de hobo.

7 L'œuvre de Beckett n'est pas sans correspondance avec le théâtre d'un Thomas Bernhard. Dans les deux cas, le monde du vaudeville, du cirque, du burlesque est la mise à nu, sans aucune forme d'embellissement, d'une «nature» humaine dont la caractéristique première est d'être sans atour.

8 Dans un autre contexte circonscrit par Widmer, la figure de Bartleby dans le récit de Melville s'impose quelques instants. Par son refus obstiné (I would prefer not to), le scribe, le commis aux écritures, se condamne peu à peu à la marginalité, au silence, à la disparition.

9 Mai 68, par exemple, avec cette image de l'enfant terrible exalté que représentait Daniel Cohn-Bendit, va susciter l'essor d'une passion, d'une soif de liberté jusqu'alors ignorée ; au Québec même, les émeutes qui eurent lieu lors des défilés de la Saint-Jean-Baptiste à la fin des années 1960 témoignaient d'une liesse que l'on voulait populaire, d'un appétit de libération, de liberté.

${ }^{10}$ De même qu'il convient de bien discerner les figures du bohémien et du vagabond d'hier, aujourd'hui du sans-abri et de l'itinérant, tant la confusion est souvent entretenue dans l'expression médiatique de la description des marginaux.

${ }^{11}$ Sur le site http://www1.bloomingdales.com/shop/search?keyword=hobo\&x=0\&y=0 (page consultée le 9 février 2015). Sinon, il y a la variante plus « hippie » et nettement moins chère : www.etsy.com/ca/market/ hobo_style_bag (page consultée le 7 janvier 2015).

12 Voir www.wikihow.com/Look-Like-a-Fashionable-Hobo ou encore, version plus abordable, www. huffingtonpost.com/2013/08/18/homeless-hobo-look-asos_n_3775716.html (pages consultées le 7 janvier 2015).

${ }^{13}$ Voir www.trendhunter.com/slideshow/hobo-fashion-features (page consultée le 7 janvier 2015).

${ }^{14}$ Voir http://www.moleskine.com/fr/news/cinelli (page consultée le 29 janvier 2015).

${ }^{15} \mathrm{Ibid}$. En complément, voir http://www.bootleg.it/hobo-moleskine/ (page consultée le 29 janvier 2015). 